\title{
Multimedia Teleservices Modelled with the OSI Application Layer Structure
}

\author{
Erwin van Rijssen \\ Logica \\ PO Box 22067 \\ 3003 DB Rotterdam \\ The Netherlands \\ ery@lbvrtda.logica.com
}

\author{
and Ing Widya \\ Centre for Telematics and \\ Information Technology \\ University of Twente \\ PO Box 217 \\ 7500 AE Enschede \\ The Netherlands \\ widya@cs.utwente.nl
}

\author{
and Eddy Michiels \\ Centre for Telematics and \\ Information Technology \\ University of Twente \\ PO Box 217 \\ 7500 AE Enschede \\ The Netherlands \\ michiels@cs.utwente.nl
}

\begin{abstract}
This paper looks into the communications capabilities that are required by distributed multimedia applications to achieve relation preserving information exchange. These capabilities are derived by analyzing the notion of 'information exchange' and are embodied in communications functionalities. To emphasize the importance of the users' view, a top-down approach is applied. The (revised) OSI Application Layer Structure (OSI-ALS) is used to model the communications functionalities and to develop an architecture for composition of multimedia services with these functionalities. This work may therefore be considered an exercise to evaluate the suitability of OSI-ALS for composition of multimedia teleservices.
\end{abstract}

\section{$1 \quad$ Introduction}

With the emergence of cheap computing power and network capacity distributed multimedia applications become feasible. These applications integrate media such as audio, video and text in order to represent information to the users in easily perceptible form [2]. The information is usually geographically dispersed and must be retrieved, processed and transmitted very fast to achieve timely delivery. The main difficulty related to the distribution of multimedia information is the preservation of relations between pieces of information. These relations may have a global perspective (e.g., simultaneous receipt of information at different destinations) and a local perspective (e.g., a time relation between subsequent samples in a video stream). To support a large variety of applications, a framework architecture is required that allows users to compose multimedia teleservices in an efficient and effective way. The architecture must provide generic communications functionalities that enable exchange of information and that preserve the relations mentioned above.

The aims of this work are the identification of some basic functional building blocks and the development of an architecture that describes how the building blocks can be composed to provide multimedia teleservices. The architecture is based on the revised OSI Application Layer Structure (OSI-ALS) [7] and therefore this work also 
examines the suitability of the OSI-ALS ${ }^{1}$ for modelling multimedia teleservices. Before a framework architecture can be defined, it must be clear what communications capabilities are required by multimedia applications. The capabilities are identified by analyzing the notion of information exchange and are quantified with QoS parameters. The top-down approach emphasizes the significant role of the users' view. Results found in the literature are used to fit this approach, see for instance $[3,10,11,16]$.

This paper is organized as follows. Section 2 derives communications capabilities that must be provided by multimedia teleservices. In section 3, a teleservice is presented that is meant to support multimedia applications like videophony. A teleservice can be decomposed into an application-oriented protocol layer and an underlying transport service. Section 4 briefly discusses the requirements a transport service must satisfy in order to be suitable for transfer of multimedia information. Section 5 elaborates a framework architecture enabling composition of multimedia teleservices on top of the discussed transport service. This architecture is validated in section 6 by modelling two important mechanisms: synchronization and QoS management. Finally, in section 7 conclusions are drawn.

\section{Communications Capabilities of Multimedia Teleservices}

A large diversity of communications capabilities is needed to satisfy the communications requirements imposed by all kinds of distributed multimedia applications. As mentioned in the previous section, communications capabilities are identified by analyzing the notion of information exchange. The term information involves the following capabilities:

- the type of information, i.e. the so-called media;

- the relations between various media information.

The term exchange involves the following capabilities:

- the communication-structure between the end-users;

- the directions of information flow in the communication structure;

- the distributed synchronism, i.e. the simultaneous submission of information from different sources or the simultaneous receipt of information at different destinations.

In the following sections, each communications capability is analyzed in more detail to identify the necessary functionalities. The functionalities are used to preserve the information characteristics and to guarantee the required relations, mentioned in section 1 . How strictly these relations must be maintained can be specified by

\footnotetext{
${ }^{1}$ At the end of this paper a list with abbreviations is added to improve the readability of this work.
} 
quantifying QoS parameters. These parameters are also derived in the following sections.

\subsection{Media}

The inter-arrival times of video information units at a destination cleary satisfy a certain regularity. A strong relation exists between the time arrivals at which successive video information-units ${ }^{2}$ are received. On the contrary, in the transfer of text information, the inter-arrival times of text information-units usually do not obey to any regularity. These examples illustrate the time relation characteristic of media information. This leads to the following definitions (see also [11]).

- Continuous Media information flow is a sequence of information-units of a single type that are related in time. Such an information flow is also called a stream. Examples are video and audio information.

- Still Media information is a set of information-units that are generally not related in time. Examples are data, text and image information.

The existence of a time relation between (uncoded) continuous media informationunits often implies some natural redundancy in the information. The higher the natural redundancy of the exchanged information, the lower the reliability of the communications service can be. The required reliability of a service can be expressed in terms of the QoS error rate parameter (see table 1).

To maintain the time relation in a stream, a minimum amount of 'bandwidth' must be continuously available. The amount of bandwidth that is needed during the use of a service can be expressed with the information-unit rate and the information-unit size parameters (see [17] and table 1).

Due to the time relation, the rate at which continuous media information-units are sent is equal, within a certain tolerance, to the rate at which they are received. In contrast, if still media information-units are transferred, different rate values at the sending and receiving sites of a communication line are conceivable. To preserve the time relation in a continuous media stream between the sending and the receiving SAPs, the QoS intra-stream jitter parameter is defined ([6] and table 1).

\subsection{Media information Relations}

This section identifies the relations that are conceivable between different media information, mentioned earlier. It also describes the functions and QoS parameters that are required to preserve the relations during information transfer.

Synchronization Relations. In a videophony application, someone's lip movements in a video scene must match with the corresponding audio information. In order to obtain 'lip-synchronization' between the individual information-units of a

\footnotetext{
${ }^{2} \mathrm{An}$ information-unit is an elementary collection of information of a particular type.
} 
video and an audio stream, the multimedia service must provide so-called continuous synchronization [3]. A necessary condition for this synchronization function is that the time between the arrival of corresponding information-units in different streams is restricted to an upperbound. This upperbound is expressed with the inter-stream jitter parameter (table 1). The synchronization that is necessary in a video play-out between control information (e.g., 'play' and 'stop') and video and audio information can be achieved by event-based synchronization [3]. It is user controllable, which means that a user can explicitly interfere each time that synchronization is required. Since it is used to express causal ordering and it is not applied in situations in which stringent time-constraints exist between information-units, the inter-stream jitter parameter is not relevant for event-based synchronization functions.

Quality Relations. Due to congestion in networks, communications service providers may not be able to maintain the required quality of a service. In this case, the provider may need a user supplied rule to enable the degradation of specific services. for this reason, the priority parameter is introduced (table 1). With this parameter, users are able to specify that they want the communications service provider to abort media information under certain circumstances. For example, in a videophony session the users may require the abortion of the video stream, as soon as the audio stream crashes.

\subsection{Communication Structures}

Th communication structure capability deals with the configuration that is provided by the communications service to enable users to communicate. Four basic communication structures can be distinguished: point-to-point, multicast, multicollect and multipoint structures (see [1] for definitions). This communications aspect does not contribute to the definition of new QoS parameters. However, in order to use these communication structures, functions that allow establishment of multiparty associations are needed.

\subsection{Communication Directions}

This capability deals with the directions in which information can be exchanged between end-users. In dialogue communications, information flows in both directions are allowed. Many distributed applications in which dialogue communications is applied have an interactive character. As a result, informationunits must be received within an acceptable time interval, in general. For example, in a videophony application this is $0.25 \mathrm{~s}$ [4]. The end-to-end delay parameter (table 1) is defined to enable users to inform the service provider about this requirement. There are also distributed multimedia applications that apply dialogue communications, but in which the end-to-end delay is not submitted to stringent upper bounds. An example is a multimedia electronic-mail service with confirmation. In some multimedia applications the information that is exchanged in both directions is of different types (e.g., a film-retrieval application), whereas in other applications the information types are equal (e.g., videophony). In the former case, the requirements imposed on the 
communications service can be quite different and must therefore be specified separately for each direction of transmission. This is also preferable in the latter case, because different end-users can have different QoS requirements.

In monologue communications the information flows in only one direction. The inability of a receiver to respond implies, in general, that the end-to-end delay requirements imposed on the communications service are less stringent than for dialogue communications.

\subsection{Distributed Synchronism}

As mentioned in the introduction of section 2, distributed synchronism is the simultaneous submission of information from different sources or the simultaneous receipt of information at different destinations, within certain human perceptible tolerance. In a 'game', e.g., all players must perceive the destruction of a target at the same moment. This requirement can be expressed with the replicated-stream jitter parameter (table 1). This parameter is therefore used to specify another aspect of information distribution in time and space. It can be considered the distributed version of the inter-stream jitter parameter.

\subsection{Overview of Quality of Service Parameters}

In table 1 an overview is shown of the QoS parameters distinguished in this paper.

End-to-End Delay: the elapsed time between offering an information-unit at the sending Service Access Point (SAP) and its delivery to the receiving SAP.

Error Rate: the estimated probability that an information-unit is subject to loss, or corruption between sending SAP and receiving SAP.

Information-Unit Rate: the rate at which information-units are sent or received at a SAP.

Information-Unit Size: the size of the information-units that are sent or received at a SAP.

Intra-Stream Jitter: the variance in information-unit inter-arrival times within a stream, measured at the receiving SAP.

Inter-Stream Jitter: the difference in inter-arrival times of synchronization-related information-units in different media information, measured at the receiving SAP.

Replicated-Stream Jitter: the difference in submission (respectively, receipt) times of information, measured at the sending (respectively, receiving) SAPs.

Priority: parameter used to distinguish between the relative importance of various media information. High priority media information are served before lower ones. Should the network become congested, lower priority information-units will be dropped before high priority information-units. 


\section{A Multimedia Teleservice}

This section elaborates a call model that provides an abstract view of conceivable communication relations between the users of a multimedia teleservice. The model includes communications capabilities (identified in previous section) that are needed to satisfy the requirements imposed by distributed multimedia applications.

\subsection{Multimedia Communications Capabilities}

With the communications capabilities mentioned below, it must be possible to support a well accepted class of distributed multimedia applications, e.g., multiparty videophony. Furthermore, users must be able to adjust the functionality of the delivered service. However, the provider of the teleservice must not be too complex to implement.

In many distributed multimedia applications two or more end-users are involved. Therefore, the teleservice must be able to provide multiparty calls [8] and support all basic communication structures (section 2.3). This service must also support continuous media information exchange (section 2.1) in interactive dialogues (section 2.4). As continuous media require a guaranteed bandwidth, the service will be connection-oriented. Moreover, this service must maintain synchronization and quality relations between media information (sections 2.2 and 2.5), necessary in applications such as multiparty conferencing.

\subsection{A Simple Call Model}

The call model describes the communications relation between users and is defined in accordance with the communications capabilities mentioned in section 3.1. The model is inspired by [5] and is discussed in more detail in [14]. This paper only discusses those issues that are relevant with respect to the evaluation of the OSI-ALS in section 6 .

First of all, a call definition specifies a set of users that are bound to the call. These users have a global call view, which consists of a global QoS view and a global communication structure view. To maintain the global call view, users are informed about all changes made to the call QoS and call communication structure. A global QoS view is necessary, because all users have to know the quality of the information that other users are able to send or receive. A global communication structure view is needed to know which users are sending information to whom.

The communication structure of a call is described as a set of service components. A service component is the basic constituent of a call (see also [5]) and models the information exchange of a single media, such as video or audio. For each service component a set of attached users is specified. The decomposition of a call into service components facilitates the adaptation to changing user needs. A call is decomposed into service components, because the end-users already distinguish between media in practice. Another advantage of treating different media as separate 
service components instead of combining them in one format is that this avoids the need for a large number of composite signals [9].

Apart from the communication structure, the QoS requirements that users impose on service components, are specified. Furthermore, the synchronization and quality relations that may exist between service components are part of the call model (see, e.g., section 2.2 and [5]). During the establishment of a call the initiating user may specify a so-called success condition. This condition specifies under which circumstances the call establishment can be considered successfully (e.g., 'user $A$ must agree with participation in the call').

Figure 1 gives an example of a call with three users. The communication structure consists of two service components: an audio component between $\mathrm{A}, \mathrm{B}$ and $\mathrm{C}$ and a video component between $\mathrm{A}$ and $\mathrm{C}$. In the rest of this paper this example is used to illustrate how the developed architecture can be applied.
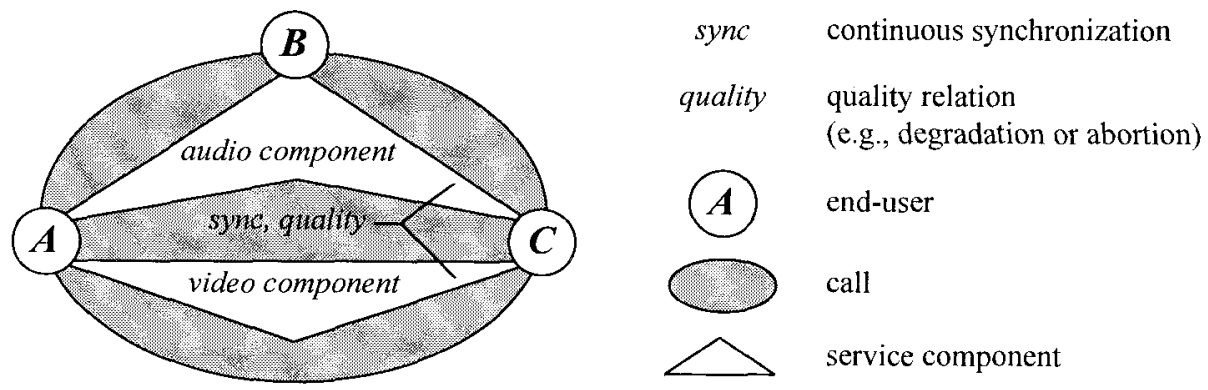

Fig. 1. Example of an Abstract View of a Multimedia Teleservice

\section{Transport Service Requirements}

Now that the communications capabilities have been defined, the service provider can be decomposed into two parts: an application-oriented protocol layer and an underlying transport service provider. To determine the functionalities of the application-oriented protocol layer, the capabilities of the transport service have to be known. In this paper, the underlying transport service is OSI Transport Service [15] look alike, in order to be compatible with the OSI-ALS. It provides end-user to enduser services. However, this service is extended with new capabilities that are easily provided by todays high speed networks, e.g., ATM-based networks.

The underlying transport service is able to provide multicasting. To support multicasting the establishment of multipoint transport connections is a necessity. Since QoS requirements can be quite different for various directions of information flow, the use of unidirectional transport connections is advocated (see, e.g., ST-II [18]). Moreover, dynamic QoS management is required for such transport connections to adapt to changing user needs (see also [3]). This means that QoS renegotiation must be possible during the information-transfer phase of a connection. 


\section{$5 \quad$ A Multimedia Framework Architecture}

A functionality gap exists between a transport service that satisfies the requirements described in previous section and the class of teleservices identified in section 3.1. This gap must be filled up with an application-oriented protocol layer. This section does not consider presentation layer functions. It develops an application-oriented architecture which enables the composition of a particular class of multimedia teleservices (see section 3.1). The aim is to develop an architecture that satisfies the following design principles:

- users are capable to tailor the teleservices to their specific needs;

- efficient and effective composition of teleservices by using predefined functional building blocks;

- extensibility of teleservices by adding or removing some building blocks.

- (re)design of teleservices, independent from the underlying transport service.

By using basic functional building blocks that are consistent with OSI-ALS, the suitability of OSI-ALS for modelling multimedia teleservices is evaluated.

First, section 5.1 presents an overview of the multimedia framework architecture. This contributes to a better comprehension of the rest of this chapter. The derivation of the architecture is divided into three steps. First, the functionalities that must be available in an application-oriented protocol layer are enumerated (section 5.2). Then, in section 5.3 three architecture levels are distinguished to facilitate the clustering of functionalities into functional building blocks. The actual clustering of functionalities into OSI-ALS consistent building blocks is performed in section 5.4. Section 5.5 shows the relations between levels, building blocks and functionalities.

\subsection{Architecture Overview}

Figure 2 shows a framework architecture that enables composition of teleservices. This architecture comprises the functional building blocks that are identified in section 5.4 .

In the world of telecommunications, a distinction is made between call control and bearer control (see, e.g., [12]).In the architecture presented in this paper a similar division can be recognized. The upper part of the architecture performs 'call control equivalent' functions, such as call establishment. In the lower part of the architecture 'bearer control equivalent' functions are available, for instance to establish the components needed for exchange of media information (e.g., video information). This separation is illustrated in figure 3 . 


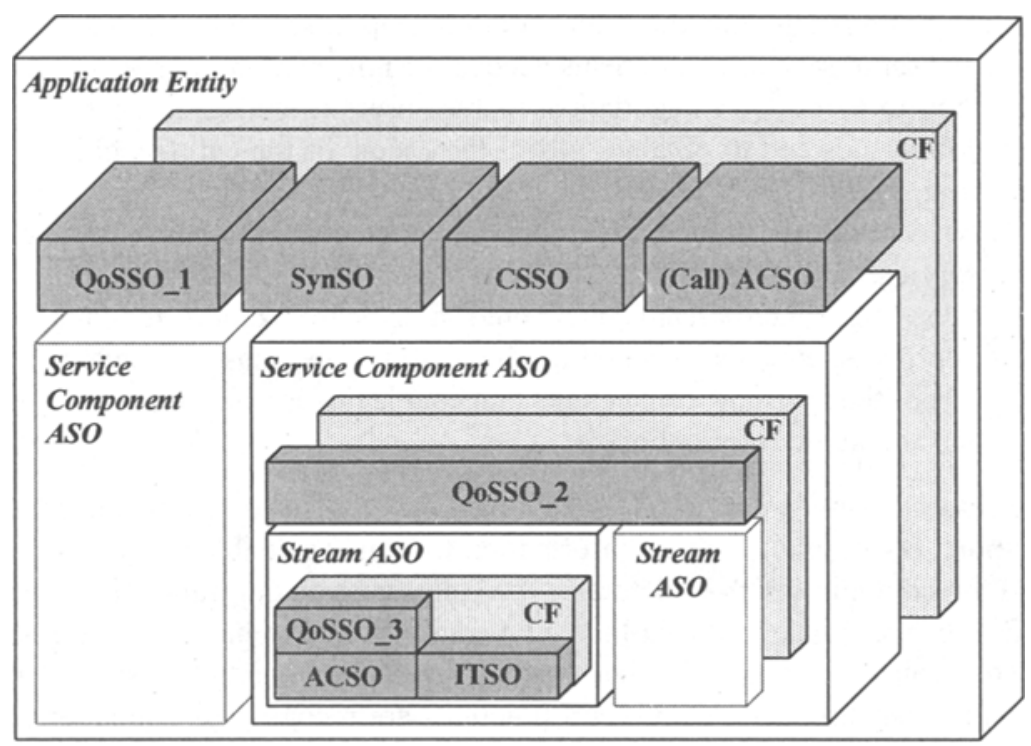

Fig. 2. Overview of the Multimedia Framework Architecture

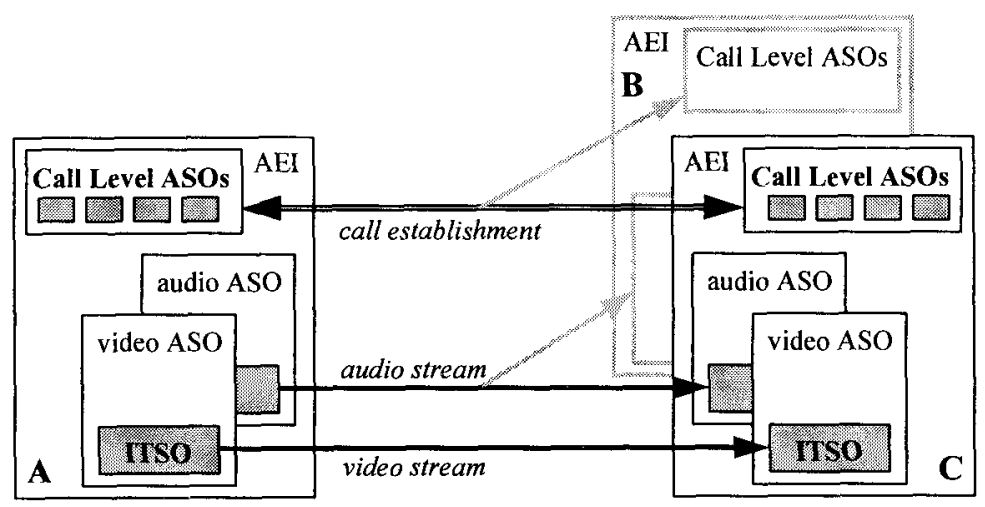

Fig. 3. Separation of 'Call Control' and 'Bearer Control'

\subsection{Architecture Functionalities}

A first step towards an application layer architecture is to determine the functionalities that are needed to provide multimedia teleservices. Since a connectionoriented teleservice is assumed (section 3.1) three phases can be distinguished during the lifetime of a call: an establishment, information-transfer and release phase.

In the establishment phase, the application layer has to perform functions such as negotiation on the call communication-structure, QoS negotiation and QoS parameter 
translation. Negotiation on the call communication-structure is performed to determine the set of service components needed for information transfer and the set of users attached to them. QoS negotiation enables users to derive an agreement from personal QoS wishes and to obtain a global QoS view of the call (section 3.2). QoS parameter translation [10] is the function that maps some of the QoS parameters of the teleservice (e.g., end-to-end delay and error rate) on those of the underlying transport service. The other QoS parameters are mapped onto application layer functionalities (e.g., inter-stream jitter onto a synchronization function). If the negotiation process appears successful, the call set-up phase is ended with the establishment of the transport connections that enable the transfer of user information, such as video and audio information.

In the information-transfer phase functionality is needed to submit information to the transport service and to receipt information from it. To enable users to modify the QoS and the communication-structure of the call, renegotiation functionality must be available. Changes made to the call must be administrated to maintain the global call view. To avoid violation of inter-stream jitter bounds and to satisfy priority requirements, synchronization and QoS functions are recognized. The latter can also be used to maintain abortion relations between service components (see section 2.2).

The release phase encompasses all functions that are needed for orderly termination of the call, such as the release of the supporting transport connections.

\subsection{Architecture Levels}

As said previously, an important requirement imposed on a multimedia framework architecture is that it must provide generic functional building blocks. In this way, teleservices can be easily composed by using predefined building blocks. Moreover, reuse of building blocks results in a cost reduction for the composition of new services. Another advantage is that users can tailor the teleservices to their specific needs by selecting building blocks from a given set and by specifying the way in which these blocks interfere. Independance from the underlying transport service is achieved by nesting of building blocks. Nesting is a new feature in the revised OSI Application Layer Structure (OSI-ALS). The size of the 'gap' between the transport service and the teleservice determines how deep the nesting is.

Functional building blocks are identified by clustering functionalities (see section 5.2) that are strongly related. In this way, each building block is associated with a particular function that is clearly defined. By assigning the clustered functionalities to the building blocks of OSI-ALS, the suitability of OSI-ALS for composition of teleservices is evaluated. In order to find an optimal clustering, it appears useful to examine how a call is mapped onto transport connections.

In section 3.2. a call is defined as a combination of service components, which are monomedia but possible bidirectional. The complexity of a call can be further reduced by dividing the service components into unidirectional, multipoint streams. Each stream corresponds to exactly one multipoint transport connection. In this way the framework architecture is structured into three levels (see also figure 2): 
- Call level;

- Service Component level;

- Stream level.

Figure 4 illustrates how the call that is shown in figure 1 is mapped onto transport connections.

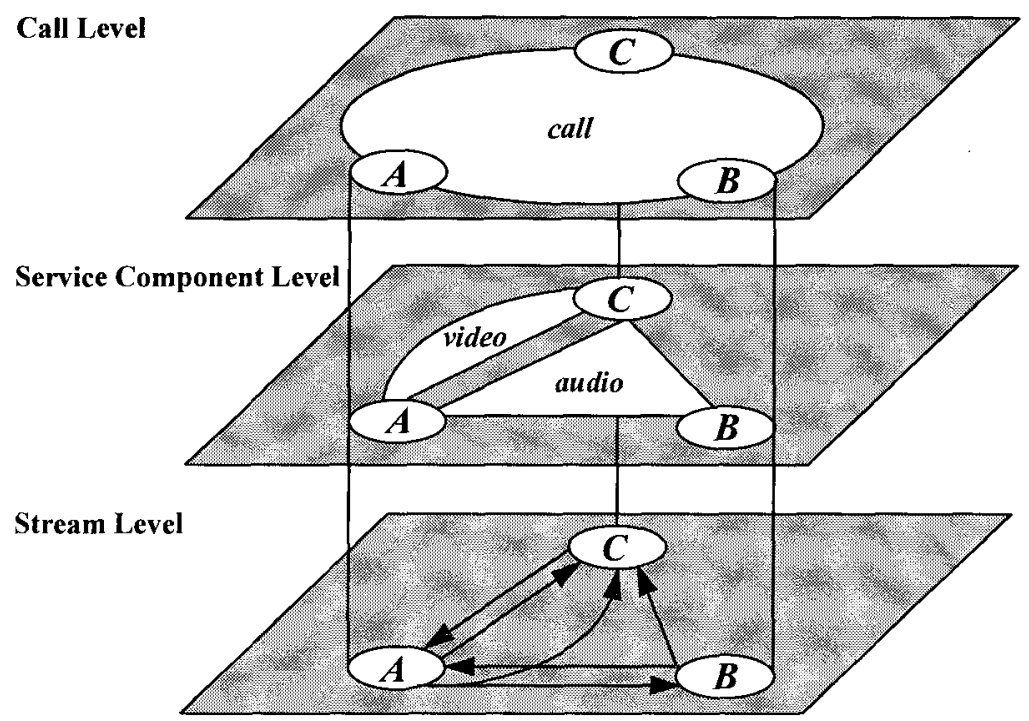

Fig. 4. Mapping a Call onto Transport Connections

\subsection{Architecture Building Blocks}

In OSI-ALS [7], Application Service Elements (ASEs) are defined as elementary building blocks. The ASEs are encompassed by other building blocks, that are called Application Service Objects (ASOs). In this paper, however, ASOs are considered the elementary building blocks and ASEs are not used. This is due to the fact that ASEs only support point-to-point communications, whereas ASOs also allow multipoint communications. In order to benefit from the multicasting capability of the transport service, the building blocks in the framework architecture must support multipoint communications. OSI-ALS allows nesting of ASOs, which satisfies the need to be able to design teleservices independent from the underlying transport service. Besides ASOs, Control Functions (CFs) are defined in the OSI-ALS. A CF is part of an ASO. It is able to control local sub-ASOs and to communicate with other (remote) CFs. It perceives all information that enters the ASO and is responsible for a correct distribution of this information over the sub-ASOs. To illustrate that CFs perceive all information, figure 2 shows them in parallel with the other ASOs. Co-ordination between peer CFs in remotely located protocol entities (Application Entity Invocations (AEIs)) is performed via the sub-ASOs. This is due to the fact that CFs in 
different AEIs cannot communicate directly (see [7]). The rest of this section explains for each level which functionalities it comprises and to which building blocks these functions are assigned.

Call Level. Before service components can be established, the users and the provider must a.o. negotiate on the call communication-structure, the call QoS and the synchronization relations between the service components. These functions are placed in a Communication-Structure ASO (CSSO), a Synchronization ASO (SynSO) and a QoS ASO (QoSSO_1), respectively (see also figure 2). To enable the negotiation between CSSOs, SynSOs and QoSSO_1s in peer Application Entities, a communication link must be set-up. This link is established by the Association Control Service Object (ACSO), which can be viewed as a multiparty extension of the OSI Association Control Service Element (ACSE) [7]. This extension also encompasses the ability to renegotiate the QoS of that link during the informationtransfer phase. The result of the negotiation procedure is determined in QoSSO_1, using the user specified success condition (see section 3.2).

During the information-transfer phase, the global communication-structure view is monitored by the CSSO. Synchronization of service components is also a function of the call level and is performed by the SynSO. Quality relations between service components (see section 2.2) are negotiated and maintained by the QoSSO_1. When a user is bound to a call, but not attached to any of the service components, the component level and the stream level in its AEI are empty. Nevertheless, this user must be kept informed about the global call view. Therefore, maintenance of the global QoS view is also assigned to QoSSO_1. The fourth function of the QoSSO_1 is call QoS renegotiation during the information-transfer phase.

The service component ASOs, that are depicted in figure 2 below the ACSO, CSSO, SynSO and QoSSO_1, can perform the actual establishment of the service components.

Service Component Level. At this level, intelligence is needed to decide how service components are decomposed into streams. This function is assigned to a $Q o S$ $A S O$ (QoSSO_2 in figure 2). It depends on the QoS requirements of the users whether multipoint or point-to-point streams (and thus transport connections) are used. For example, if one user wants to receive video information of HDTV quality, while for another user normal quality is sufficient, QoSSO_2 must decide whether the video information is multicast over a single multipoint transport connection or transmitted over separate point-to-point transport connections. In the former case the information is submitted once, thereby satisfying the quality requirements of the most demanding user. In the latter case the same information is submitted twice to the transport service, but with different QoSs. QoSSO_2 may also decide to split the video component into, e.g., two streams. Hereby, one stream has a more reliable QoS than the other, to transfer the coded video information-units that are sensitive to errors. The other stream transfers the less error sensitive part of the coded video information.

Stream Level. QoS negotiation which is necessary at this level, is restricted to the transport connections that have to be established. A QoS ASO ( $Q O S S O \_3$ in figure 2) 
is used for this purpose. It also maps most application-oriented QoS parameters (e.g., end-to-end delay) onto transport connection parameters (e.g., transit delay). To exchange the media information-units (e.g., video-information units), InformationTransfer ASOs (ITSOs in figure 2) are used. The stream level ACSO establishes the transport connections that are needed to transfer the media information-units.

\subsection{Relations between Levels, Building Blocks and Functionalities}

This section presents an overview of the relations between levels in the architecture, the building blocks and the functionalities these building blocks perform.

\begin{tabular}{|c|c|c|}
\hline Level & ASOs & Functionalities \\
\hline Call & $\begin{array}{l}\text { QoSSO_1 } \\
\text { SynSO } \\
\text { CSSO } \\
\text { (Call) ACSO } \\
\text { SC ASOs }\end{array}$ & $\begin{array}{l}\text { QoS (re)negotiation \& maintenance } \\
\text { Maintenance of global QoS view } \\
\text { Negotiation \& maintenance of synchr. relations } \\
\text { Comm.-structure (re)negotiation \& maintenance } \\
\text { Maintenance of global comm.-structure view } \\
\text { Establishing link via which peer QoSSO_1s, } \\
\text { SynSOs and CSSOs communicate } \\
\text { (zero or more) }\end{array}$ \\
\hline$S C$ & $\begin{array}{l}\text { QoSSO_2 } \\
\text { Stream ASOs }\end{array}$ & $\begin{array}{l}\text { Deciding (based on QoS) whether multicast or } \\
\text { point-to-point transport connections are used } \\
\text { (one or more) }\end{array}$ \\
\hline Stream & $\begin{array}{l}\text { QoSSO_3 } \\
\text { ACSO } \\
\text { ITSO }\end{array}$ & $\begin{array}{l}\text { QoS } \\
\text { Establishing \& releasing transport connections } \\
\text { Submission/receipt of information to/from transport } \\
\text { connections }\end{array}$ \\
\hline
\end{tabular}

Table 2. Relations between Levels, Building Blocks and Functionalities

\section{Evaluation of the Framework Architecture}

In section 5.1 an architecture for composition of multimedia teleservices was presented. In order to evaluate the suitability of the architcture, section 6 selects two mechanisms and shows how they can be modelled with the functionalities defined in the architecture. A more complete evaluation would result in too much detail for this paper. The mechanisms selected are synchronization and QoS management. Synchronisation is discussed because it is often applied to satisfy the requirements 
that the end-users impose on the distributed multimedia applications (see section 2). QoS management is illustrated bcause a lot of advanced distributed multimedia applications require a flexible QoS that can easily be adapted to changing user needs.

\subsection{Synchronization}

Many synchronization mechanisms can be distinguished, but in this paper only two, frequently applied mechanisms are discussed. The first mechanism is marker synchronization. Hereby, markers are placed in the user information to obtain synchronization between related user information-units. The second mechanism that is dealt with is channel synchronization. This mechanism uses a control channel alongside the user information channels [16].

- Marker Synchronization. When synchronization markers are used, the synchronization information is exchanged over the same transport connections as the user information. Therefore, the SynSO of the call level inserts the markers between the user information-units. This is done via the CFs of the service component level (see figure 5a). As a consequence, the ITSOs at the stream level must submit two types of Protocol Data Units (PDUs): synchronization PDUs and user information PDUs (e.g., video PDUs). A SynSO negotiates beforehand with SynSOs in peer AEIs about the synchronization mechanism to be used. This is indicated by the thin dashed arrow under the SynSO in figure 5a. Notice that for simplicity purposes figure 5 represents the CFs in another way than figure 2 .

- Channel Synchronization. When channel synchronization is applied, the external synchronization channel transfers tuples of identifiers of information-units that have to be synchronized. In this case, the SynSO does not submit synchronization information to the CFs of the service component level (see figure 5b). Instead, the synchronization information is exchanged between peer AEIs via a special transport connection. To enable the SynSO to establish the needed transport connection, the SynSO is refined with an internal ACSO. This is possible since the OSI-ALS allows the nesting of ASOs.

Figure 5 shows how the functionalities of the framework architecture can be composed to provide synchronization. The architecture allows enough flexibility to model both discussed synchronization mechanisms. Furthermore, a close look at figure 5 teaches that the structure of the AEI hardly changes when a teleservice is extended with new service components that have to be synchronized as well. This indicates that the architrecture allows easy extension of teleservices, even when a complex mechanism as synchronization is involved. 


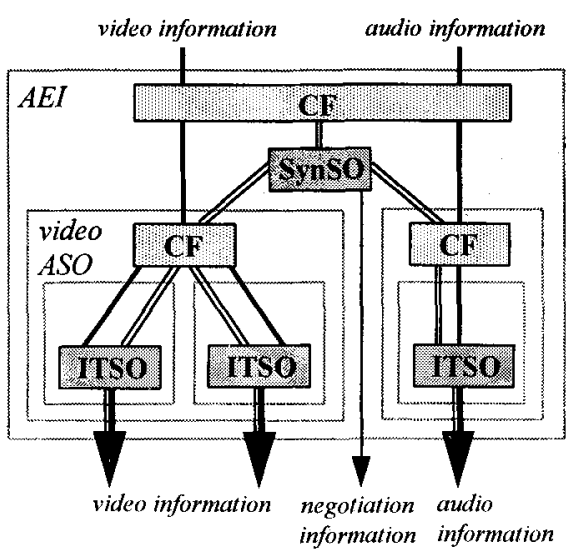

a) Marker Synch ronization

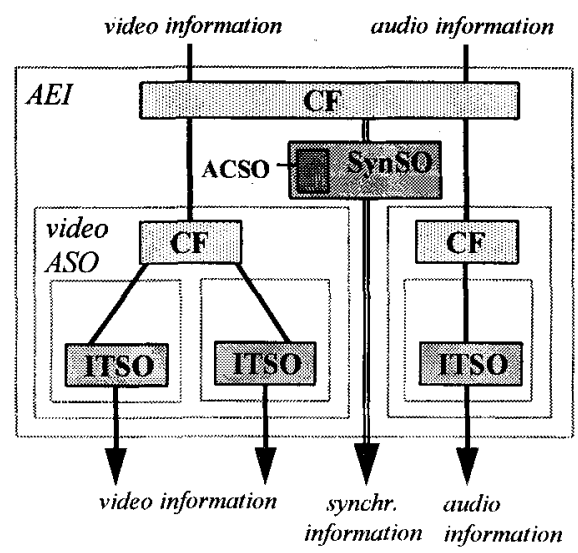

b) Channel Synchronization

Fig. 5. Application Entity Invocations for different Synchronization Mechanisms

\subsection{Quality of Service Management}

QoS management comprises several functions, such as QoS (re)negotiation and QoS maintenance.

- QoS Renegotiation. Due to changing user needs, QoSSO_1 may be requested to perform QoS renegotiation. The result of the renegotiation procedure is sent by QoSSO_1 to QoSSO_2 (see the dashed line of figure 6 between these ASOs). Then, QoSSO_2 can examine whether the new QoS differs so much from the old one that splitting (or integration) of transport connections is required. Moreover, it can examine the need for QoS renegotiation on existing transport connections. In case this appears necessary, QoSSO_2 asks QoSSO_3 to perform this QoS renegotiation (see the dashed line in figure 6 between these ASOs).

- QoS Maintenance. The QoS of a transport connection is monitored by the QoSSO 3 of the stream ASO that corresponds to that connection. When the obtained QoS does not satisfy the requirements, QoSSO_3 can start a QoS renegotation procedure. If this procedure fails, QoSSO_3 may decide to release the transport connection, which is then performed by ITSO. Another kind of QoS management is the surveillance of the quality relations that may exist between different service components. For example, in a videophony session it is conceivable that the users require the abortion of the video component when the audio component has crashed. QoSSO_1 surveys whether this quality relation is still satisfied. Once it notices that the audio service component is crashed, it indicates to all ACSOs in the video component (via QoSSO_2 and QoSSO_3) that the video transport connections must be released as well.

The architecture offers enough flexibility to model QoS management, since changing QoS needs do not result in complicatd changes to the AEI. The modularity that is obtained by using OSI-ALS consistent building blocks makes it possible to compose an AEI with which QoS management can be tailored to specific user needs. 


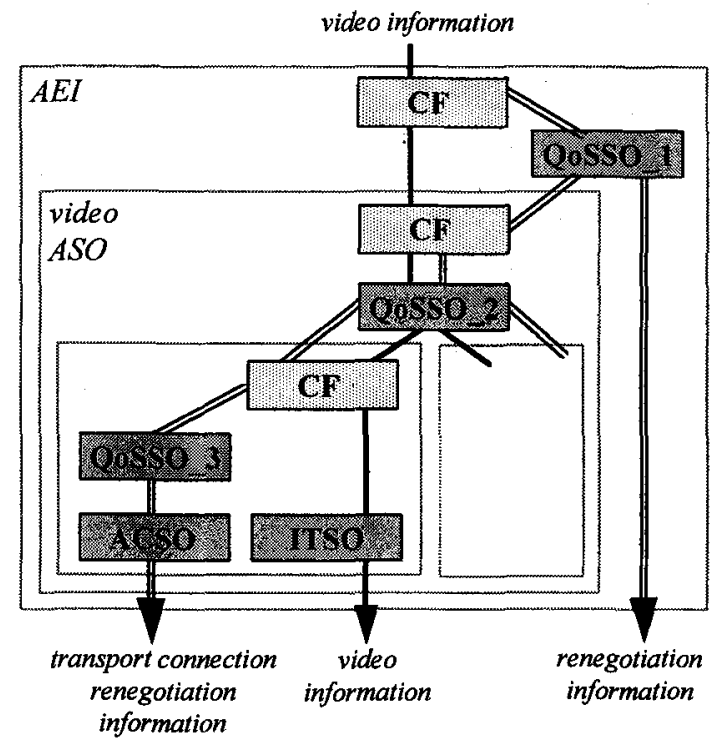

Fig. 6. Application Entity Invocation for QoS Management

Figure 7 shows how the $\mathrm{CF}$ at the call level distributes control information over SynSO, QoSSO 1 and CSSO in order to manage a video and an audio ASO. The call control information is sent to peer AEIs over the link that is denoted with the dashed line in the figure. During the call establishment phase, before the negotiation on the QoS and call communication-structure is finished, the video and audio do not yet exist. In this phase, only the call control link is present to enable the negotiation procedures.

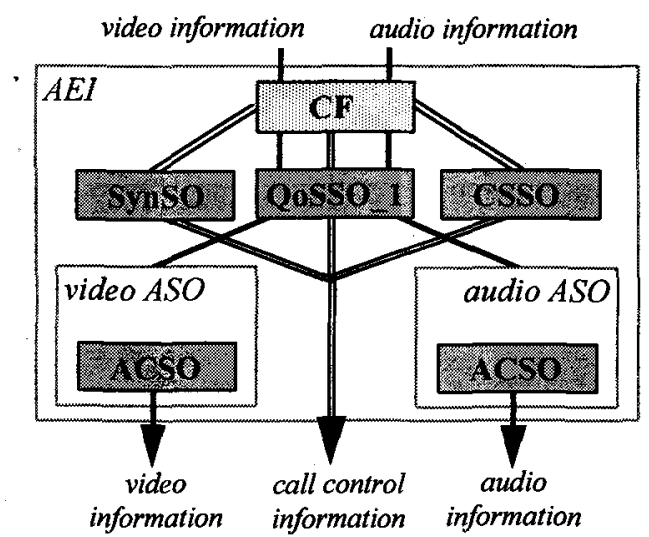

Fig. 7. Application Entity Invocation for Control of Video and Audio ASOs 


\section{Conclusions}

This paper derives in a top-down approach the communications capabilities required by distributed multimedia applications. These capabilities are embodied in functionalities such as synchronization and maintenance of quality relations, that are quantified with application-oriented QoS parameters The functionalities are clustered into functional building blocks, that are defined in an architecture that allows a flexible composition of teleservices. Most application-oriented QoS parameters are mapped onto QoS parameters of an underlying transport service. OSI-ALS is used successfully to elaborate a multimedia framework architecture. This exercise therefore shows to some extent the suitability of OSI-ALS to model multimedia teleservices. However, the composition rules prescribed in OSI-ALS give also rise to some questions. For example, it is not clear why peer CFs in different AEIs have to communicate via underlying ASOs instead of communicating directly.

The elaborated architecture, which consists of three levels, indicates that the nested structure of OSI-ALS provides independence from an underlying transport service. The Application Service Objects (ASOs), defined in OSI-ALS, are appropriate to serve as elementary building blocks of the architecture, since they enable effective and efficient composition of user tailorable teleservices (see the requirements mentioned in section 5). The Association Control Service Object (ACSO), which establishes multiparty associations to exchange information, is expected to be easily realizable with a new transport service like ST-II [18] or the transport service provided by XTP [13]. Notice however that XTP does not support any QoS yet.

Finally, it is worthwhile to remark that the elaborated example shows an architecture which has several similarities with architectures found in the telecommunications area, although the OSI-ALS originates from the OSI datacommunications area.

\section{Acknowledgement}

The authors would like to thank Geert Heijenk and Xinli Hou for the intensive discussions on the call model, and Dick Quartel and Robert Huis in 't Veld for their contributions to the first two sections.

\section{Glossary}

ACSE Association Control Service Element

ACSO Association Control Service Object

AEI Application Entity Invocation

ASE Application Service Element

ASO Application Service Object

ATM Asynchronous Transfer Mode

CF Control Function 


$\begin{array}{ll}\text { CSSO } & \text { Communication-Structure Service Object } \\ \text { ITSO } & \text { Information-Transfer Service Object } \\ \text { OSI } & \text { Open Systems Interconnection } \\ \text { OSI-ALS } & \text { OSI Application Layer Structure } \\ \text { PDU } & \text { Protocol Data Unit } \\ \text { QoS } & \text { Quality of Service } \\ \text { QoSSO } & \text { QoS Service Object } \\ \text { SAP } & \text { Service Access Point } \\ \text { SC } & \text { Service Component } \\ \text { SynSO } & \text { Synchronization Service Object }\end{array}$

\section{References}

1. S.R. Ahuja and J.R. Ensor: Coordination and Control of Multimedia Conferencing. IEEE Communications Magazine, May 1992, pp. 38-43.

2. S.A. Bly, S.R. Harrison and S. Irwin: Media Space: Bringing People together in a Video, Audio and Computing Environment. Communications of the ACM 36(1), January 1993, pp. 28-47.

3. A. Campbell, G. Coulson, F. Garcia and D. Hutchison: A Continuous Media Transport and Orchestration Service. Sigcomm '92, 1992, pp. 99-110.

4. D.B. Hehmann, M.G. Salmony and H. Stuttgen: Transport Services for Multimedia Applications on Broadband Networks. Computer Communications 13(4), May 1990, pp. 197-203.

5. G.J. Heijenk, X. Hou and I.G. Niemegeers: Service Description of Communications Systems Supporting Multi-media Multi-user Applications. IEEE Network, January 1994.

6. ISO: Quality of Service Framework - Working Draft No. 1, November 1992. ISO/IEC JTC1/SC21 N7521.

7. ISO: Final Text of ISO/IEC 9545 Application Layer Structure, May 1993. ISO/IEC JTC1/SC21 N7815.

8. S.E. Minzer: A Signaling Protocol for Complex Multimedia Services. IEEE Journal on Selected Areas in Communications 9(9), December 1991, pp. 1383-1394.

9. S.E. Minzer and D.R. Spears: New Directions in Signaling for Broadband ISDN. IEEE Communications Magazine 27(2), February 1989, pp. 6-14.

10. K. Nahrstedt and J. Smith: Revision of QoS Guarantees at the Application/Network Interface. Technical Report MS-CIS-93-34, University of Pennsylvania, March 1993. 
11. C. Nicolaou: An Architecture for Real-Time Multimedia Communications Systems. IEEE Journal on Selected Areas in Communications 8(3), April 1990, pp. 391-400.

12. A. Paglialunga and M. Siviero: ISCP (ISDN Signalling Control Part): the Best Candidate for the Target Broadband-ISDN Signalling Protocol. Communication Networks 4(2), April 1993, pp. 193-200.

13. Protocol Engines, Inc.: XTP Protocol Definition, Revised 3.4, July 1989.

14. E.H. van Rijssen: A Survey of Distributed Multimedia Requirements for Application Layer Structuring. Master's Thesis, University of Twente, Department of Computer Science, Enschede, The Netherlands, October 1993

15. SIS Standardiseringsgrupp: Information Processing Systems - Open Systems Interconnection - Connection-oriented Transport Service Definition, ISO 8072-1986, December 1986.

16. R. Steinmetz: Synchronization Properties in Multimedia Systems. IEEE Journal on Selected Areas in Communications 8(3), April 1990.

17. R. Tokuda, Y. Tobe, S.T.-C. Chou and J.M.F. Moura: Continuous Media Communication with Dynamic QOS Control Using ARTS with an FDDI Network. Sigcomm '92, August 1992, pp. 88-99.

18. C. Topolcic: Experimental Internet Stream Protocol, Version 2 (ST-II). Internet Request for Comments No. 1190, RFC-1190, October 1990. 